\title{
Adaptive Architecture, an Implementation with Game Theory. Emotional Input and Pneumatically Driven Actuator
}

\author{
Yann Blanchi ${ }^{1}$, Corinne Touati ${ }^{2}$, Elizabeth Mortamais ${ }^{3}$ \\ ${ }^{2}$ INRIA Grenoble, France \\ 1yann.blanchi@paris-valdeseine.archi.fr \\ ${ }^{2}$ corinne.touati@inria.fr \\ 3emortamais@gmail.com
}

${ }^{1,3}$ EVCAU Laboratory, School of Architecture of Paris Val de Seine, France

\begin{abstract}
In the active context of adaptive architecture, this proposal tackles the field of Human Building Interaction. Indeed, material would no longer be inert but activated by the users, programmable and equipped with memory. The information chain penetrating each component of the house forms a field of globalities. On the one hand, in line with the under development of smart materials, giving intelligence to previously passive materials, we consider the pneumatically driven actuator material as soft robot and programmable and on the other hand, we implement swarm communications with means of innovative IT elements. Since the elements of the smart material are interdependent, we consider a novel approach of modeling their interactions, using tools from the vast field of game theory. The digital information travels gradually through the physical material. In a technical point of view, first, we expect that a pneumatic cells network could be considered as a Human Building Interaction. HBI could operate using both remote and haptic information, one constituted by emotional records, the other reacting to the physical contact. We focus on the emotional implementation and the haptic inputs, within a pneumatic cells network actuator. We considered both, inhabitant emotion and kinesthetic communication as inputs and we implemented pneumatically driven actuators. The pneumatic cells network is used as a soft changing interface, a dynamic architecture that links the building with the inhabitants, an architectural apparatus that supports an active process in a changing way. A program that can be described as a multi-player game addresses the pneumatic cells network.
\end{abstract}

Keywords: Human building interaction; Game theory; Pneumatic cells network 